J. Lake Sci.(湖泊科学), 2019, 31(2): 517-528

DOI 10. 18307/2019. 0220

(C) 2019 by Journal of Lake Sciences

\title{
基于无人机和卫星遥感影像的升金湖草滩植被地上生物量反演”
}

\author{
高 燕, 梁泽毓 ${ }^{1}$, 王 彪 ${ }^{1,2}$, 吴艳兰 ${ }^{1,2 * *}$, 刘诗雨 ${ }^{2}$ \\ ( 1 : 安徽大学资源与环境工程学院, 合肥 230601) \\ ( 2 : 安徽省高分辨率对地观测系统安徽数据与应用中心, 合肥 230001)
}

\begin{abstract}
摘 要: 湿地植被地上生物量是衡量湿地生态系统健康状况的重要指标,对于珍稀水禽越冬繁殖、全球碳循环、生态净化 具有重要意义, 是生态学与遥感解译的研究热点之一. 针对于地上生物量的测算, 卫星遥感数据覆盖范围广但其空间分 辨率较低, 无人机遥感数据空间分辨率高但采集范围小, 同时受湿地面积、观测系统及外界环境等条件的影响, 使得遥感 影像地上生物量反演更加复杂和困难. 本研究基于无人机和高分一号数据对升金湖草滩植被地上生物量反演进行研究, 结合升金湖保护区 4 个样区无人机可见光影像与相应样区实测样本数据, 建立地上生物量与可见光波段、多种可见光植 被指数的线性、幂函数、多项式、对数回归模型, 并通过可决系数 $\left(R^{2}\right)$ 、平均绝对误差 $(M A E)$ 和均方根误差 $(R M S E)$ 对模 型进行精度评价, 选择最优模型对无人机影像进行地上生物量反演; 通过可见光波段反演得到的生物量, 与高分一号 WFV 归一化差分植被指数 (Normalized Difference Vegetation Index, NDVI) 影像相结合进行回归建模, 获取整个升金湖草滩 植被地上生物量分布. 结果表明, 利用无人机红光波段建立的多项式方程对地上生物量反演有着最高模拟精度, $R^{2}=$ 0.86 、预测精度 $M A E=111.33 \mathrm{~g} / \mathrm{m}^{2} 、 R M S E=145.42 \mathrm{~g} / \mathrm{m}^{2}$, 且红光波段生物量反演方法得到的结果与实际生物量分布一致 性较高, 高分一号 WFV NDVI 与无人机反演生物量构建的多项式模型为最优模型, $R^{2}$ 为 0.91 . 本研究利用无人机和高分 一号数据进行生物量反演研究, 整合多源遥感数据优点, 以获取更加丰富和准确的信息, 进而提高地上生物量反演精度, 为湿地监测和湿地恢复管理提供数据和技术支撑,具有重要研究意义和应用价值.
\end{abstract}

关键词: 湿地; 地上生物量反演; 无人机遥感; 高分一号;植被指数; 升金湖

\section{UAV and satellite remote sensing images based aboveground biomass inversion in the meadows of Lake Shengjin}

\author{
GAO Yan ${ }^{1}$, LIANG Zeyu ${ }^{1}$, WANG Biao ${ }^{1,2}$, WU Yanlan ${ }^{1,2 * *} \&$ LIU Shiyu ${ }^{2}$ \\ (1: School of Resources and Environmental Engineering, Anhui University, Hefei 230601, P.R.China) \\ (2: Anhui Data and Application Center of High Resolution Earth Observation System in Anhui, Hefei 230001, P.R. China)
}

\begin{abstract}
The aboveground biomass of wetland vegetation, as an essential indicator of the wetland ecosystem health, is of great significance for the overwintering reproduction, global carbon cycle and ecological purification of rare waterfowl. It is one of the research hotspots in ecology and remote sensing interpretation. The advantage of satellite remote sensing data lies in its wide coverage, but its spatial resolution is low. UAV remote sensing data have high spatial resolution but small acquisition range. At the same time, because of the influence of wetland area, observation system and external environment, it is more complicated and difficult to retrieve the aboveground biomass from remote sensing images. This research studies a kind of inversion method of aboveground biomass based on UAV and GF-1 data. Firstly, UAV visible images of four sample areas and the ground measured sample data are used to establish linear, power function, polynomial, and logarithmic regression model of biomass, visible light band, and a variety of visible light vegetation index. The accuracy of this method was evaluated by the coefficient of determination $\left(R^{2}\right)$, mean absolute error $(M A E)$ and root mean square error (RMSE). The optimal model was selected for biomass inversion of UAV images. Then the biomass data inverted from the visible light band and the GF-1 WFV normalized difference vegetation index (NDVI) image are used
\end{abstract}

* 安徽省教育厅重点项目 (KJ2018A0007) 、安徽省人力厅留学人员创新项目择优资助计划和安徽省国土资源厅科技 项目 (2016KJ030002) 联合资助. 2018-05-11 收稿; 2018-08-23 收修改稿. 高燕 (1992 ), 女, 硕士研究生; E-mail:gaoyan_ahu@163.com.

** 通信作者; E-mail: wuyanlan@ ahu.edu.cn. 
to establish a regression model to obtain the aboveground biomass distribution map of the vegetation in Lake Shengjin meadows. The results show that the polynomial equation was determined using the red band has higher simulation accuracy for biomass inversion, $R^{2}=0.86, M A E=111.33 \mathrm{~g} / \mathrm{m}^{2}, R M S E=145.42 \mathrm{~g} / \mathrm{m}^{2}$, and the inversion results obtained by the red band biomass inversion method is highly consistent with the actual biomass distribution. The polynomial model, constructed by GF-1 WFV and biomass inversed by UAV, is the optimal model, and $R^{2}$ reached 0.91 . This study uses UAV and GF-1 data to conduct biomass inversion research. It integrates the advantages of each data and can obtain richer and more accurate information. It could improve inversion accuracy and provide data and technical support for wetland monitoring and wetland restoration management. Thus this work has important research significance and application value.

Keywords: Wetlands; aboveground biomass inversion; UAV; GF-1; vegetation index; Lake Shengjin

湿地植被生物量, 是衡量湿地生态系统健康状况的重要指标, 作为湿地生态系统固炭能力的一个重要 参数, 在全球碳循环中发挥着重要的作用 ${ }^{[1-2]}$. 传统的湿地植被生物量测算方法主要有样方调查和收割法 等 ${ }^{[3]}$, 但传统调查方法进行大范围湿地生物量研究时, 人力物力耗费巨大, 在空间全局性、时效性等方面存 在着明显的局限性, 并且会对调查区域内植被造成严重破坏 ${ }^{[4]}$. 而遥感数据则可以弥补这些不足, 可为湿地 植被生物量反演提供大量有用的信息 ${ }^{[5-6]}$. 众多学者从不同的光学遥感数据 ${ }^{[1]}$ 、反演模型 ${ }^{[5,7]}$ 、植被指数 ${ }^{[8-10]}$ 等方面进了湿地生物量遥感反演研究.

随着遥感技术的发展, 利用多源遥感数据互补性和合作性协同反演植被地上生物量已成为必然趋势. 目前利用多源遥感数据反演湿地植被地上生物量的研究较少, 一般是根据研究目的和研究区域特殊的环境 特点及植物类型, 选择不同的遥感数据进行生物量反演进而提高精度 ${ }^{[11]}$. 例如: 针对湿地水环境对光学遥 感反演生物量的影响及对植被高度的考虑, 将光学遥感和雷达数据相结合反演湿地生物量以提高反演精 度 ${ }^{[12]}$; 利用中等空间分辨率 Landsat 数据和高时间分辨率 MODIS 数据融合得到高时空分辨率的 NDVI 数据 反演草原生物量 ${ }^{[13]}$. 目前生物量反演仍是以卫星遥感作为主要数据源, 而无人机遥感技术作为卫星遥感的 重要补充手段, 以其机动性、时效性、经济性等优势, 近年来成为国内外学者争相研究的热点问题 ${ }^{[14-15]}$. 但由 于湿地的难进人性, 利用无人机进行植被生物量反演多是针对草地 ${ }^{[16]}$ 、林地 ${ }^{[17-18]}$, 对湿地植被生物量进行反 演及应用. ${ }^{[19]}$ 的研究较少. 另外, 无人机多用来做精度评价, 例如利用无人机影像计算的植被覆盖度和生物 量作为真值, 对 SPOT6 影像与实测生物量数据构建的反演模型进行精度评价 ${ }^{[20]}$, 这也间接说明无人机遥感 反演生物量的可靠性.

在利用卫星遥感数据反演生物量时, 生物量像元真值获取通常情况是在均一地表中, 取像元对应范围 内若干个样方的生物量均值作为像元生物量的 “相对真值” ${ }^{[16]}$. 但在实际应用中, 像元对应范围内植被生长 状况不尽相同, 以若干个样方的生物量均值作为像元生物量的 “相对真值” 会有偏差; 另一方面在中低分辨 率卫星遥感影像中会存在混合像元的情况, 也会造成一定的反演误差, 但卫星遥感数据可提供大范围的湿 地植被地表覆盖信息. 相比较之下, 无人机遥感空间分辨率可达厘米级, 易于获取像元生物量真值, 且能够 显著降低混合像元对反演精度的影响. 然而, 无人机遥感影像覆盖范围有限, 难以满足大面积湿地植被生物 量研究. 因此, 结合卫星和无人机遥感各自优势进行生物量反演, 可以获得更可靠的生物量像元真值及大范 围的生物量分布.

综上考虑, 本研究以地面实测鲜重生物量为无人机像元真值, 构建与无人机可见光波段及可见光波段 植被指数的回归模型, 选择最优回归模型对样区无人机影像进行生物量反演; 再以无人机反演生物量为高 分一号影像像元真值, 与 GF-1 WFV 的 NDVI 构建生物量回归模型, 并选择最优模型对高分一号影像进行生 物量反演,分析和讨论整个研究区域地上生物量的空间分布情况.

\section{1 研究区及数据来源}

\section{1 研究区域概况}

升金湖自然保护区 $\left(30^{\circ} 15^{\prime} \sim 30^{\circ} 30^{\prime} \mathrm{N}, 116^{\circ} 55^{\prime} \sim 117^{\circ} 15^{\prime} \mathrm{E}\right)$ 位于安徽省池州市境内, 以升金湖为中心, 沿 岸分别向外延伸 $2.50 \mathrm{~km}$ 左右, 自西向北分为上、中、下湖区, 总面积 $333.40 \mathrm{~km}^{2}$, 是国家级重点水禽自然保 护区和重要湿地保护区 ${ }^{[21-22]}$. 升金湖通过黄湓闸北接长江, 为季节性浅水通江湖泊, 丰水期升金湖湖面面积 
达到 $140 \mathrm{~km}^{2}$, 枯水期可降至 $34 \mathrm{~km}^{2}$, 暴露出大面积草滩, 为东方白鹳、白顶鹤、豆雁等珍稀鸟类提供了良好 的生存环境与食物来源,成为其重要的越冬栖息地 ${ }^{[23-24]}$. 升金湖自然保护区 2005 年加人 “东亚一澳大利西亚 涉禽保护区网络”, 是国际重要湿地之一 ${ }^{[25]}$, 对升金湖保护区内草滩植被生物量进行及时、有效的监测, 可 以为升金湖生态环境管理与珍稀鸟类保护工作提供有力的数据支撑. 本文研究区范围为去除保护区内基本 不受升金湖水位影响的城镇、林地和耕地等区域后, 分布于上湖、下湖及湖周围的草滩(图 1a), 草滩植被以 苔草群落为主.

\section{2 数据来源及处理}

1.2.1 生物量实测数据 由于升金湖草滩范围较大, 而无人机单次航测范围有限, 同时, 升金湖草滩空间异质 性较小, 样地内植被主要是以苔草植被为主, 以及少量的廖科植被. 为使样方更具代表性, 2017 年 3 月 8 10 日, 选择升金湖上湖四个地形平坦样区, 共 68 个样方进行苔草滩植被生物量数据采集, 此时苔草群落处 于生长阶段, 植被高度约 1 20 cm. 在每个样区选择均一地表随机布设数个 $0.4 \mathrm{~m} \times 0.4 \mathrm{~m}$ 的样方, 研究区域 及采样区域如图 1. 齐地面收割样方内的所有植被, 进行装袋标记称重, 统计湿生物量, 此生物量为样方总生 物量. 在 $0.4 \mathrm{~m} \times 0.4 \mathrm{~m}$ 的均一样区内, 草滩空间异质性小, 为与无人机像元大小保持一致性, 取样方内像元平 均生物量 (即样方内总生物量与像元总数的比值) 进行回归建模. 此外, 考虑到无人机遥感影像空间位置信 息存在误差, 尤其是受风力等外界环境因素影响, 本研究在进行无人机数据采集前, 先进行样方布设,待无 人机数据采集结束后再进行样方采集, 避免由于影像与实际地物之间的空间位置不对应而产生的反演误差. 1.2.2 无人机遥感影像获取及处理 本研究采用的是多旋翼小型无人机, 全球定位系统 (GPS)/全球导航卫 星系统 GLONASS 双模卫星定位. 无人机配备 $1 / 2.3$ 英寸 1240 万像素 CMOS 影像传感器. 可以获取红、绿、蓝 真彩色 JPEG 格式影像, 像幅 4000×3000 像素. 根据样区选择, 规划航线, 设置航向重叠度 $80 \%$, 旁向重叠度 $70 \%$, 飞行高度 $100 \mathrm{~m}, 4$ 个样区具体的天气状况和飞行范围见表 1 . 获取无人机影像后, 分区域生产样区正 射影像, 主要过程分为 3 个步骤: (1) 导人无人机影像及其对应位置信息; (2) 点云加密及匹配, 并在进行空中 三角测量处理时对影像数据进行标准化预处理, 避免后期数据处理过程中由于影像获取时间差异所造成反 演误差; (3) 检验空三数据质量结果, 生成数字表面模型 (DSM) 和正射影像, 地面空间分辨率 $4 \mathrm{~cm}, 4$ 个样区 无人机真彩色影像如图 $1 \mathrm{~b}$, 影像叠加在高分一号 WFV 真彩色影像上.

1.2.3 GF-1 WFV 影像获取及处理 考虑到样本采集时间和卫星遥感影像的质量, 本文选用了 2017 年 2 月 27 日的云含量为 $0 \%$ 的高分一号 WFV 卫星多光谱遥感影像. 该影像空间分辨率为 $16 \mathrm{~m}$, 宽幅 $800 \mathrm{~km}$, 包括 红、绿、蓝、近红外 4 个波段. 首先对多光谱影像通过辐射定标和大气校正模型, 将波段像素值由 DN (Digital Number) 值转变为辐射值, 消除遥感图像中由大气散射引起的辐射误差. 然后利用升金湖保护区矢量文件对 获取的影像进行裁剪,如图 1a. 并结合无人机正射影像对其进行几何校正,均方根误差为 0.43 .

表 1 升金湖无人机数据采集详情

Tab.1 Data specifications of UAV data collection in Lake Shengjin

\begin{tabular}{cccccc}
\hline 时间 & 天气 & 样区编号 & 航测范围 $/ \mathrm{m}^{2}$ & 样方数 & 照片数量 \\
\hline $2017-03-08$ & $3 \sim 17^{\circ} \mathrm{C}$, 晴, 东北风 1 级 & A & 295800 & 20 & 237 \\
$2017-03-09$ & $5 \sim 21^{\circ} \mathrm{C}$, 多云, 西北风 1 级 & B & 367200 & 10 & 411 \\
$2017-03-10$ & $10 \sim 18^{\circ} \mathrm{C}$, 小雨, 东北风 1 级 & C & 336000 & 20 & 155 \\
& & D & 457500 & 18 & 188 \\
\hline
\end{tabular}

\section{2 研究方法}

\section{1 无人机生物量反演}

2.1.1 可见光波段植被指数选取 植被指数是对地表植被活动简单、有效和经验的度量, 它可以有效地反映 植被健康与覆盖信息, 已作为一种有效的遥感数据处理手段, 被广泛应用于土地覆盖变化检测、植被类型识 别和生物量估测等方面. 目前, 有 150 多种植被指数被提出, 但大多是可见光和近红外波段进行不同组合形 成的, 如归一化植被指数 $N D V I^{[26]}$. 基于可见光波段植被反射率研究, 国内外学者构建了一些可见光植被指 
(a) 升金湖GF-1 WFV假彩色影像

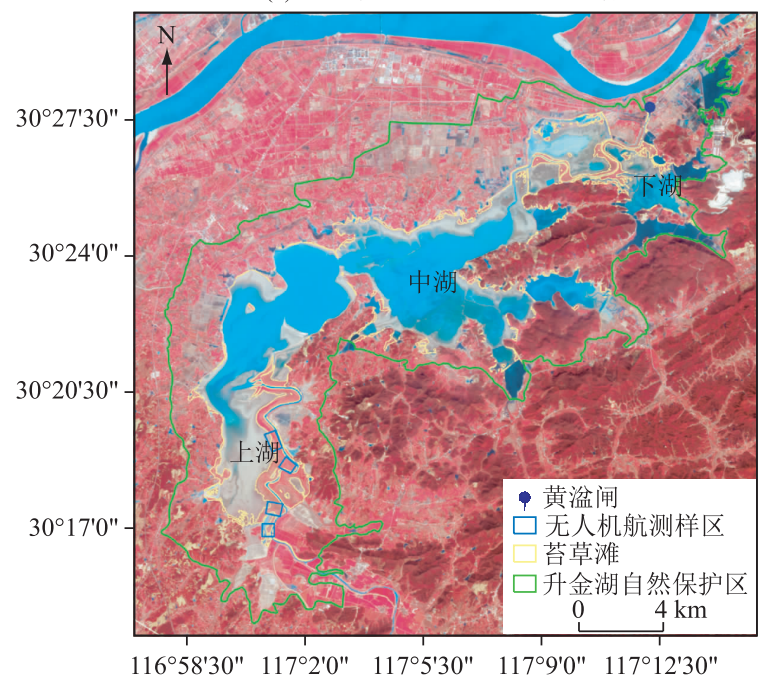

(b) 无人机航测样区影像

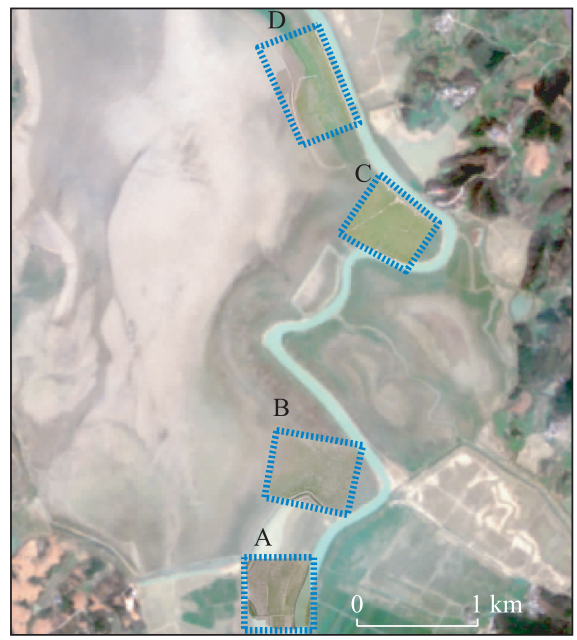

图 1 升金湖自然保护区及无人机遥感数据采集区域

Fig. 1 Lake Shengjin Nature Reserve and UAV remote sensing data acquisition area

数, 并在蓝藻识别、草地生物量反演、植被信息提取等研究中得到应用 ${ }^{[16,26-28]}$, 如表 2 列举了主要的可见光植 被指数,其中 red、green、blue 分别表示红、绿、蓝波段.

表 2 主要可见光植被指数

Tab.2 Primary visible vegetation index

\begin{tabular}{lcc}
\hline 植被指数 & 计算公式 & 文献 \\
\hline 红绿比值指数 RGRI & $\frac{\text { red }}{\text { green }}$ & {$[29]$} \\
归一化绿红差异指数 NGRDI & $\frac{\text { green-red }}{\text { green }+ \text { red }}$ & {$[30]$} \\
可见光波段差异植被指数 VDVI & $\frac{2 g r e e n-(\text { red }+ \text { blue })}{2 g r e e n+(\text { red }+ \text { blue })}$ & {$[27]$} \\
红绿蓝植被指数 RGBVI & $\frac{\text { green }{ }^{2}-(\text { red } \times \text { blue })}{\text { green }{ }^{2}+(\text { red } \times \text { blue })}$ & {$[31]$} \\
归一化绿蓝差异指数 $N G B D I$ & $\frac{\text { green-blue }}{\text { green }+ \text { blue }}$ & {$[32]$} \\
\hline
\end{tabular}

2.1 .2 反演模型的建立和精度评价 按照无人机正射影像中样方大小及编号构建矢量面文件, 并将生物量湿 重对应录人到属性信息中, 计算得到样方对应无人机影像像元的均值、最大值、最小值, 本文用像元均值进 行回归建模. 随机选择实测样本总量的 70\% (48 个), 利用回归拟合方法分别构建像元平均生物量与无人机 可见光波段及 5 种可见光波段植被指数间的线性、幂函数、多项式、对数回归模型.

本文通过可决系数 $\left(R^{2}\right)$ 、平均绝对误差 $(M A E)$ 和均方根误差 $(R M S E) 3$ 个指标对无人机生物量反演模 型进行精度评价. $R^{2}$ 是判断回归模型拟合程度优劣最常用的数量指标, $R^{2}$ 值越接近 1 , 表明拟合程度越 好 ${ }^{[33]} ; M A E$ 和 $R M S E$ 能衡量预测值与测量值之间的偏差, 其值越小, 预测模型精度越高, 本文将样本数据的 $30 \%$ (20 个) 用于计算 $M A E$ 和 $R M S E$ 做精度评价. $R^{2} 、 M A E$ 和 $R M S E$ 的计算公式分别如下: 


$$
\begin{gathered}
R^{2}=1-\frac{\sum_{i=1}^{n}\left(y_{i}^{0}-\hat{y}_{i}\right)^{2}}{\sum_{i=1}^{n}\left(y_{i}^{0}-\bar{y}\right)^{2}} \\
M A E=\frac{\sum_{i=1}^{n}\left|y_{i}-y_{i}^{0}\right|}{n} \\
R M S E=\sqrt{\frac{\sum_{i=1}^{n}\left(y_{i}-y_{i}^{0}\right)^{2}}{n}}
\end{gathered}
$$

式中, $y_{i}$ 为验证样本数 $i$ 预测值, $y_{i}^{0}$ 为样本 $i$ 的测量值, $\bar{y}$ 为样本测量值均值, $n$ 为验证样本数.

\subsection{GF-1 生物量反演}

2.2.1 反演模型的建立 考虑到 GF-1 影像存在混合像元的情况, 且像元对应范围内的生物量实测数据较难 获取, 在本研究中, 我们将无人机反演生物量作为 GF-1 影像的像元生物量真值, 与 GF-1 WFV NDVI 影像构 建生物量反演模型. NDVI 是植被生长状态和空间分布的最佳指示因子, 因其计算简单、检测灵敏, 对植被检 测幅度较宽, 有较好的时空适应性, 与植被分布密度有很好的相关性, 因此广泛应用于遥感植被监测 ${ }^{[34-35]}$. 通过公式(4)计算 GF-1 WFV 影像归一化植被指数 $N D V I$, 其中 $n i r$ 表示近红外波段.

$$
N D V I=(n i r-r e d) /(n i r+r e d)
$$

GF-1 生物量反演模型的构建步骤具体如下: 首先随机建立 50 个与 GF-1 影像像元大小位置对应的 $16 \mathrm{~m} \times 16 \mathrm{~m}$ 面矢量数据, 并统计对应的高分一号 $N D V I$ 影像像元值, 录人到属性信息中; 其次综合考虑 $R^{2} 、$ $M A E 、 R M S E$, 选择最优的无人机影像与生物量拟合方程, 计算得到四个无人机航测样区生物量空间分布图. 统计 50 个面矢量数据对应的无人机反演总生物量, 录人到属性信息中; 最后利用回归拟合方法构建 GF-1 $\mathrm{WFV}$ 影像 NDVI 值与无人机反演生物量的线性模型、幂函数模型、多项式模型、对数模型.

2.2.2 精度评价 在建立的 4 种 GF-1 生物量回归模型中, 根据 $R^{2}$ 选择最优模型, 对整个升金湖草滩植被进 行生物量反演, 得到生物量分布图, 并将本文方法与卫星遥感方法进行比较. 在本文中, 卫星遥感方法是基 于 GF-1 WFV NDVI 影像和实测 48 个采样数据, 分别构建线性模型、幂函数模型、多项式模型、对数模型, 选 择最优模型反演升金湖草滩植被生物量, 得到生物量分布. 为了验证这两种方法的准确性, 我们以无人机反 演结果为参考, 随机选择 20 个 $16 \mathrm{~m} \times 16 \mathrm{~m}$ 样方, 计算 $M A E 、 R M S E$ 进行定量评价.

\section{3 结果与分析}

在无人机红、绿、蓝波段及植被指数与实测生物量数据构建的线性、幂函数、多项式、对数回归模型中, 多项式回归模型对应的 $R^{2}$ 最高为数据拟合最优模型, 其结果如图 2. 可以看出, 红、绿、蓝波段及 $R G R I$ 与实 测生物量均为负相关关系, $N G R D I 、 V D V I 、 R G B V I 、 N G B D I$ 与生物量为正相关关系. 其中实测生物量与红波段 构建的多项式回归模型 $R^{2}=0.86$ 明显大于其他回归模型, 样本散点均匀分布在趋势线两侧, 波段反射值对 应的生物量振幅区间小. 其次是 $R G R I$ 和 $N G R D I, R^{2}$ 分别为 0.48 和 0.47 . 再者是 $V D V I$ 和 $R G B V I, R^{2}$ 分别为 0.44 和 0.43 . 之后是绿波段 $\left(R^{2}=0.32\right)$ 、蓝波段 $\left(R^{2}=0.35\right)$. NGBDI 的复相关系数是最低的为 $R^{2}=0.25$.

通过观察表 2 中的公式, 可以发现 $R G R I$ 与 $N G B D I$ 是由红和绿波段组合计算得到, $V D V I$ 和 $R G B V I$ 是由 红、绿、蓝波段组合计算得到的, $N G B D I$ 是由绿、蓝波段组合计算得到的. 因此由相同可见光波段组合计算的 植被指数反演湿地苔草滩植被生物量具有相似的效果; 红波段与实测生物量相关性最高, 而绿、蓝波段对于 草滩植被不敏感, 在生物量反演中反而使得模型精度下降. 一方面这是由于无人机遥感影像植被反射率主 要来源于自身吸收特性, 受大气散射等因素的影响较小, 且植物叶绿素吸收红光, 叶面在可见光红波段有很 强的吸收特性; 另一方面实验区域地表植被覆盖主要为苔草, 受异类地物干扰较小, 且湿地特殊的水环境, 使得湿地植被与其他地表覆盖类型在红波段的反射率差异大于蓝绿波段, 从而减少了裸地等地表覆盖类型 的影响提高了反演精度.

图 3、图 4 分别是无人机生物量反演模型的验证样本实测值一预测值散点图和基于红波段的 D 样区无 

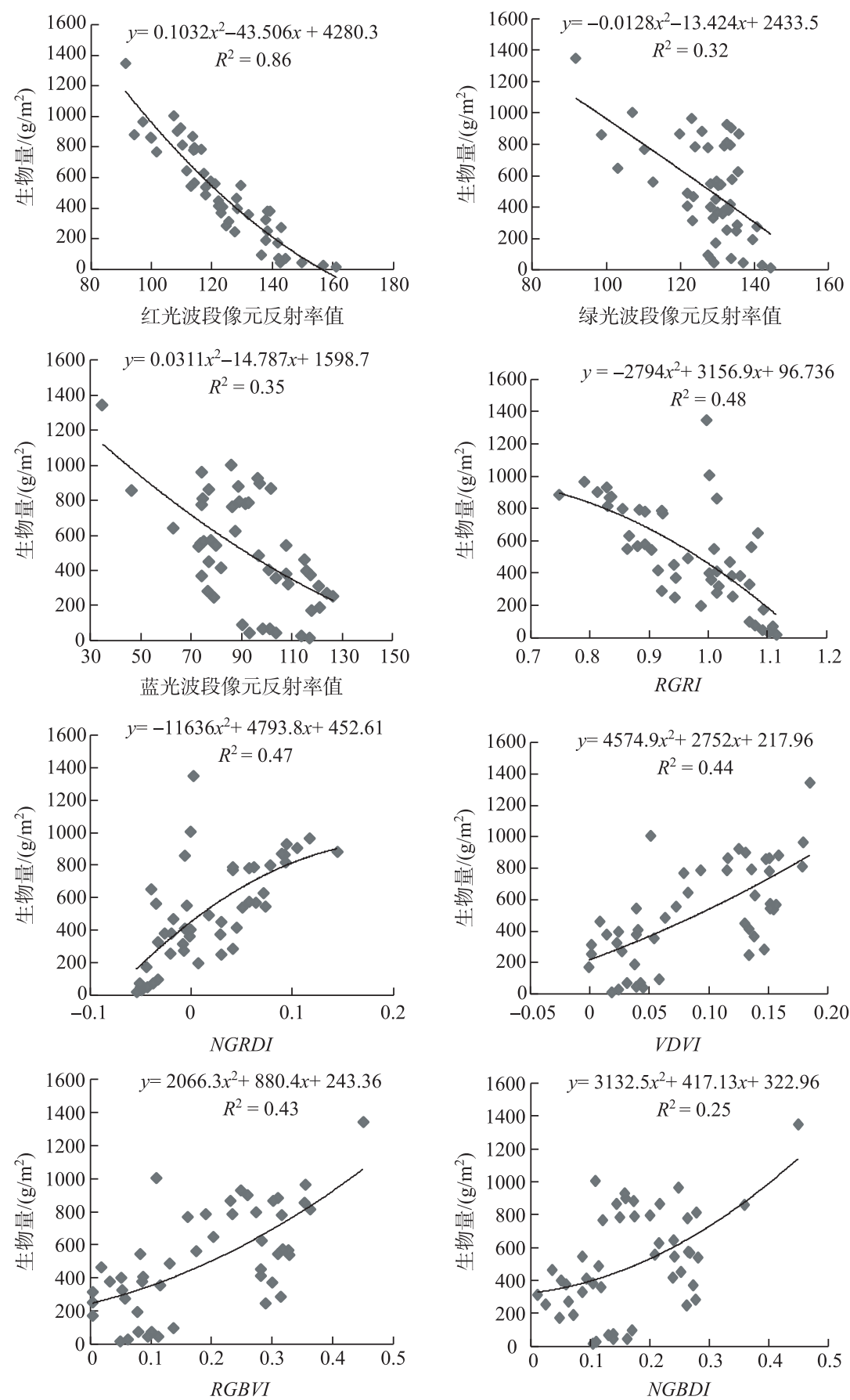

图 2 生物量与无人机可见光波段及可见光植被指数间的相关性分析

Fig. 2 Correlation analysis between biomass and UAV visible band and visible vegetation index

人机地上生物量反演结果. 可以看出红波段与生物量回归模型中 $M A E=111.33 \mathrm{~g} / \mathrm{m}^{2}$ 和 $R M S E=145.42 \mathrm{~g} / \mathrm{m}^{2}$ 值最小, 并且无人机生物量反演结果与实际情况一致性较强,有植被区域与无植被区域界限分明, 不同长势 的植被覆盖范围清晰. 因此, 基于红波段多项式回归模型的预测效果较好且较稳定. 从图 $4 \mathrm{~b}$ 和 $4 \mathrm{c}$ 可以看出 
(a) 红光波段模型

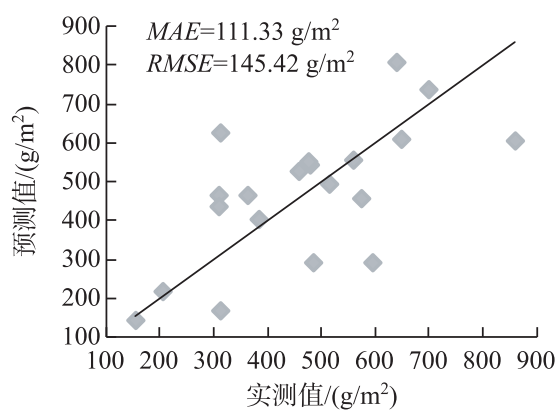

(c) 蓝光波段模型

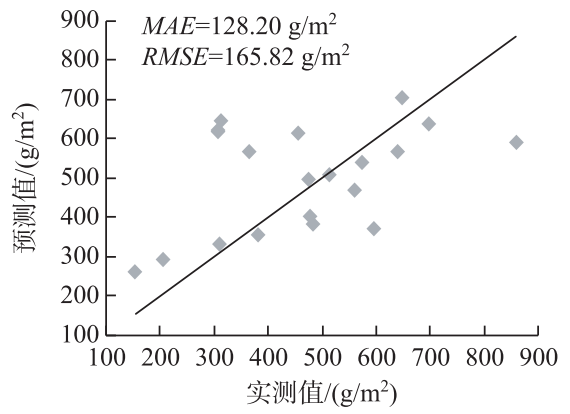

(e) NGRDI模型

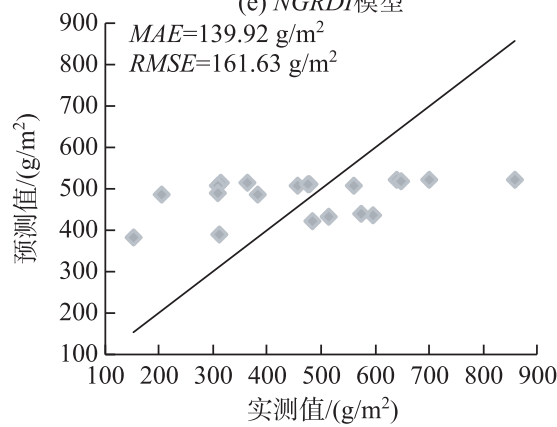

(g) $R G B V I$ 模型

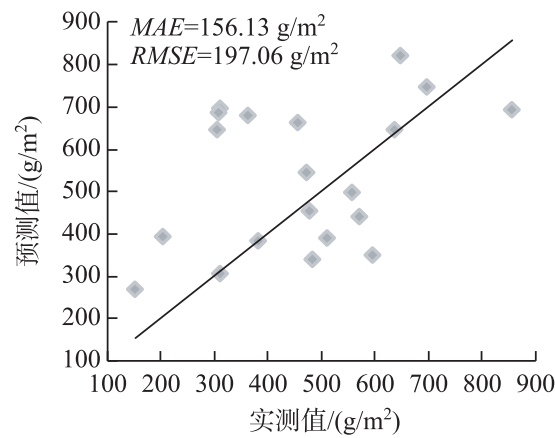

(b) 绿光波段模型

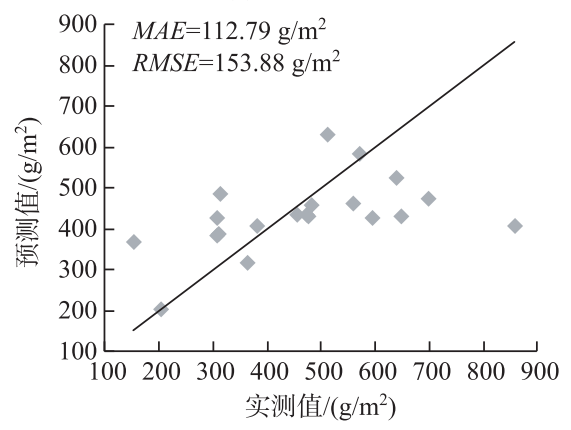

(d) RGRI模型

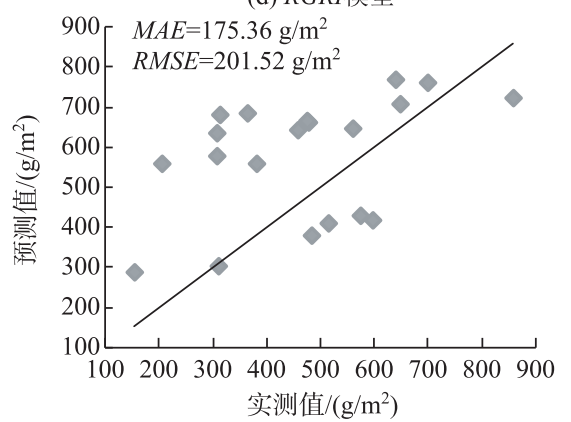

(f) $V D V I$ 模型

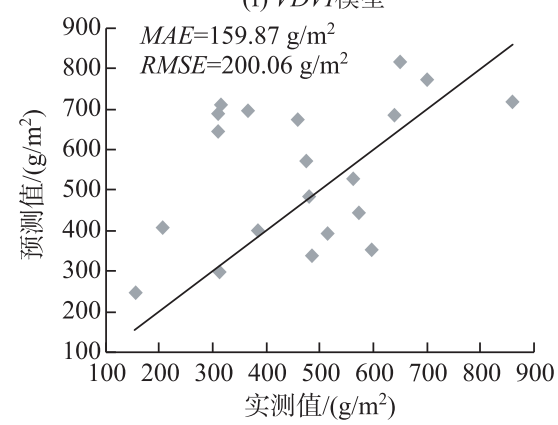

(h) NGBDI模型

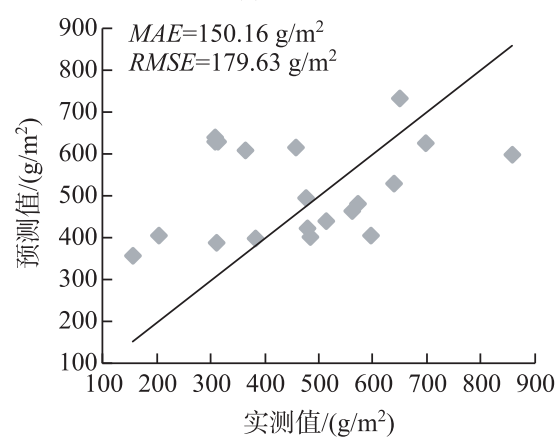

图 3 无人机生物量反演模型的 $M A E$ 和 $R M S E$

Fig. 3 MAE and RMSE for UAV biomass inversion models 
(a) D样区无人机真彩色影像

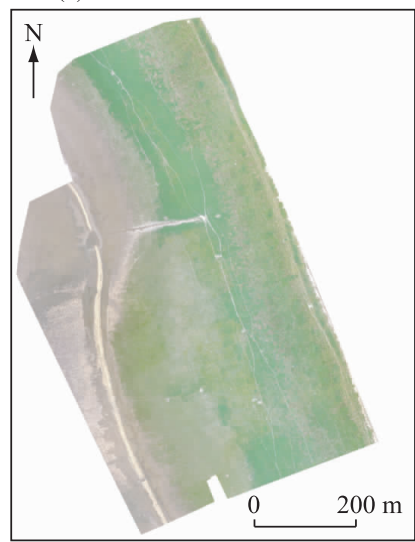

(b) D样区无人机生物量反演结果影像

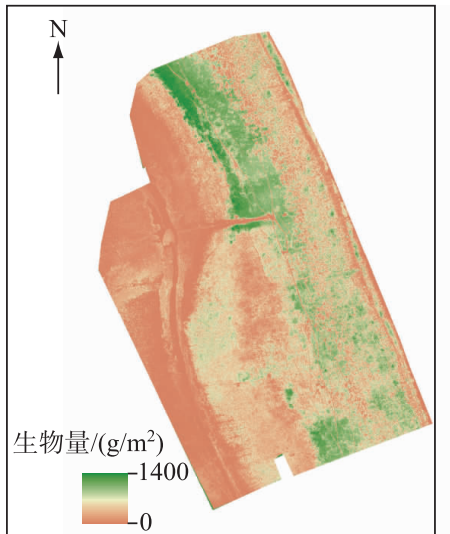

(c) D样区GF-1 WFV NDVI影像

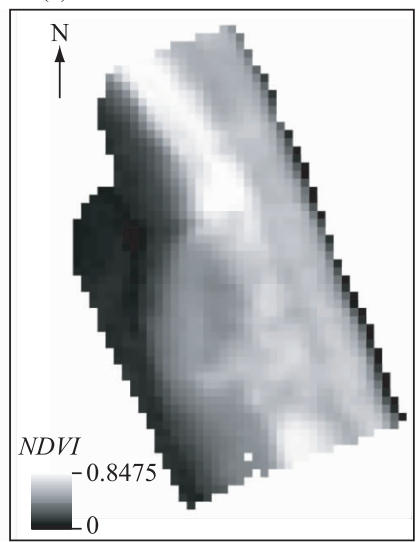

图 4 基于 red 波段的 D 样区无人机地上生物量反演结果

Fig.4 Aboveground biomass inversion results of UAV images based on red band in D-sample region

$\mathrm{D}$ 样区无人机地上生物量反演影像与 GF-1 WFV NDVI 影像具有较高的植被分布一致性.

在基于 GF-1 WFV NDVI 指数与无人机反演植被地上生物量构建线性模型、幂函数模型、多项式、对数模 型中,多项式模型的 $R^{2}=0.91$ 最大, 如图 5 所示. 样本散点均匀分布在趋势线两侧, 拟合相关性较好. 因此, 选择此模型对升金湖保护区草滩植被地上生物量进行大范围遥感反演, 并按 10 个等级进行划分), 可以看 出升金湖草滩主要分布在上湖和下湖区域 (图 6a), 在湖边会有小面积的零星分布. 生物量在 179.73 $1472.61 \mathrm{~g} / \mathrm{m}^{2}$ 之间, 下湖及其湖边的生物量主要集中分布在 $179.73 \sim 488.57 \mathrm{~g} / \mathrm{m}^{2}$ 之间, 而上湖的生物量主 要分布在 $488.57 \sim 1472.62 \mathrm{~g} / \mathrm{m}^{2}$ 之间.

(a) 多项式回归模型

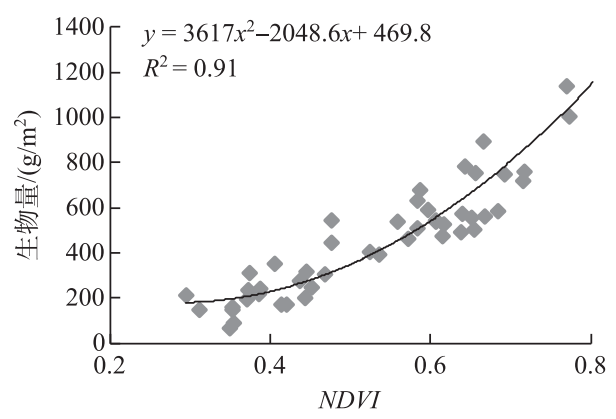

(c) 对数回归模型

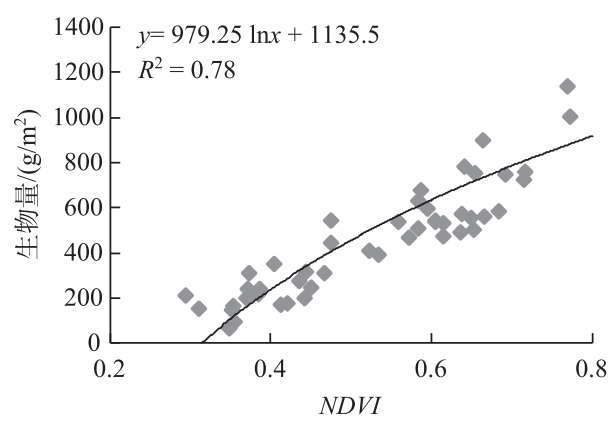

(b) 线性回归模型

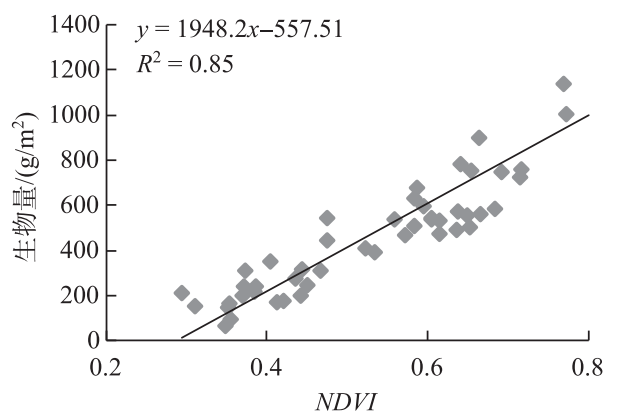

(d) 幂回归模型

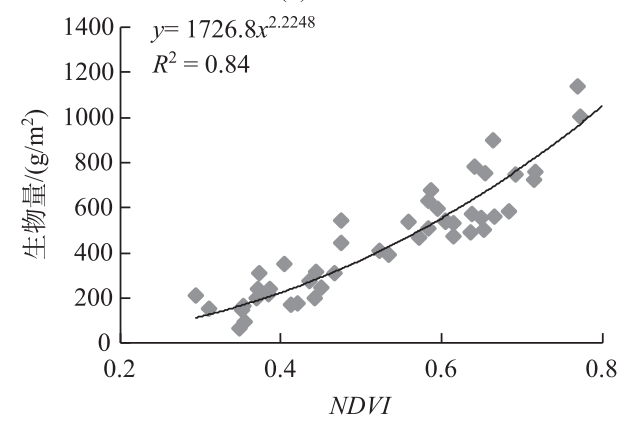

图 5 无人机反演生物量与 GF-1 WFV NDVI 的相关性分析

Fig.5 Correlation analysis of UAV inversion biomass and GF-1 WFV NDVI 
在基于 GF-1 WFV NDVI 影像和实测样方数据构建的线性模型、幕函数模型、多项式模型、对数模型中, 幂函数模型 $\left(y=2517.4 x^{2.8857}\right)$ 的 $R^{2}=0.51$ 最大, 因此, 选择此模型进行生物量遥感反演, 并按 10 个等级进行 划分, 如图 6b. 卫星遥感方法反演生物量最小值为 $46.09 \mathrm{~g} / \mathrm{m}^{2}$, 最大值为 $1746.84 \mathrm{~g} / \mathrm{m}^{2}$, 与本文方法的反演结 果在整个升金湖草滩生物量分布趋势上一致. 为了进一步更精确的比较本文方法与卫星遥感方法的反演结 果, 以无人机反演结果数据为参考比较了两种方法在 D 样区的反演结果如图 7 及计算的 $M A E 、 R M S E$ 如图 8 . 可以看出, 两种方法与无人机反演结果相比, 相同位置下两种方法生物量反演精度存在较大差异, 卫星遥感 方法反演结果偏大, 且 $M A E 、 R M S E$ 值都大于本文反演结果, 特别是在一些植被长势差异大或不同地物类型 边界处 (图 7 黑框处). 这主要是由于卫星遥感方法反演时是以像元对应范围内若干个样方的生物量均值作 为像元生物量的 “相对真值”, 当影像存在混合像元时导致反演结果偏大. 本文方法以无人机反演结果作为 介质数据可以有效地提高反演精度,降低混合像元对反演精度的影响.

(a) 本文反演结果

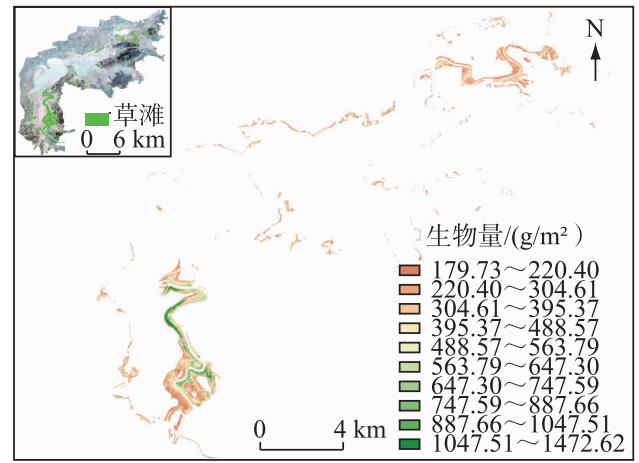

(b) 卫星遥感反演结果

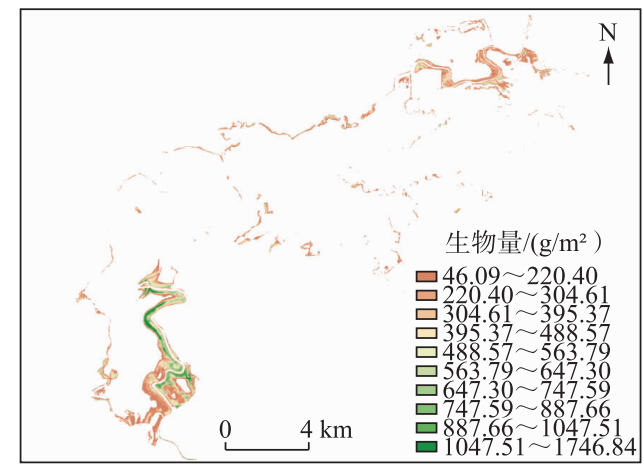

图 6 升金湖草滩地上生物量 GF-1 WFV 反演结果

Fig. 6 Inversion results of GF-1 WFV in the meadows aboveground biomass of Lake Shengjin

(a) D样区无人机反演结果

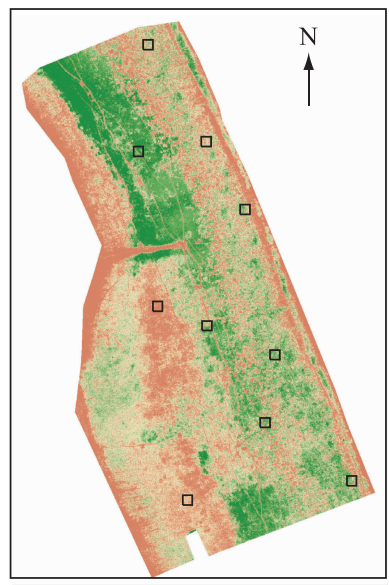

(b) D样区本文方法反演结果

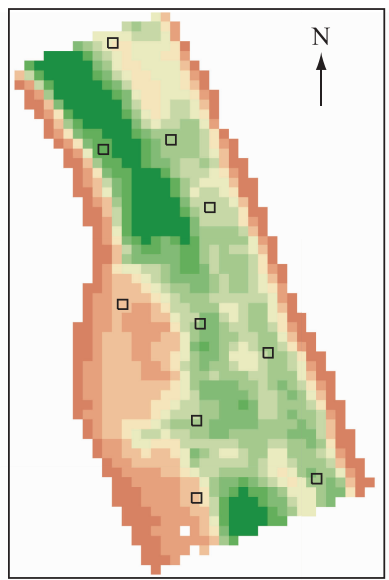

(c) D样区卫星遥感方法反演结果

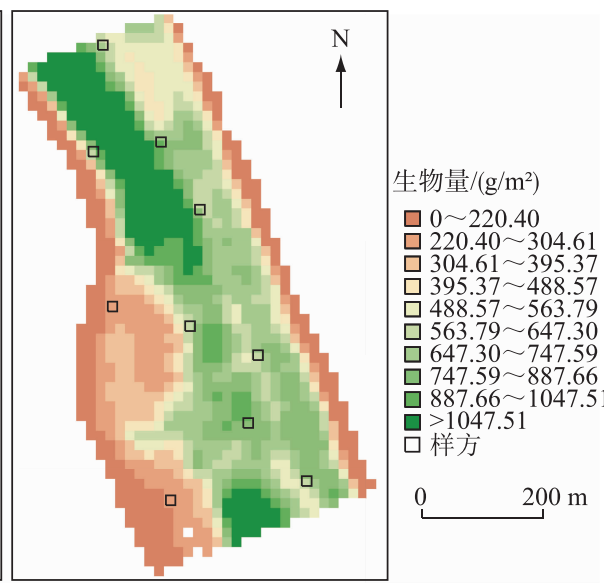

图 $7 \mathrm{D}$ 样区生物量反演结果比较

Fig.7 Comparison of biomass inversion results in D-sample region

本文提出方法反演生物量最低值为 $179.73 \mathrm{~g} / \mathrm{m}^{2}$, 大于无人机反演和卫星遥感反演结果. 一方面, 本文方 法在构建 GF-1 生物量回归模型时, 以无人机生物量反演均值作为其所在像元生物量, 对模型结果精度造成 一定损失. 另一方面, 无人机生物量和 GF-1 生物量反演结果, 受到混合像元和多项式构建过程中误差的影 
响, 部分偶然误差累积为系统误差, 也会对最后反演结果产生有一定影响. 然而, 相比于卫星遥感反演方法, 本文提出方法反演生物量具有明显优势, 可以有效提高湿地地上生物量反演的精度和效率, 极大减少外业 样本采集数量. 且无人机的机动性、时效性、经济性以及 GF-1 的时间分辨率高、覆盖范围广等优势使得本研 究方法具有更广的适用性. 虽然本文方法可以获得较高的反演精度,但本文反演模型较为简单,通过考虑环 境因素构建多因素反演模型和结合混合像元分解方法可进一步提高模型精度.

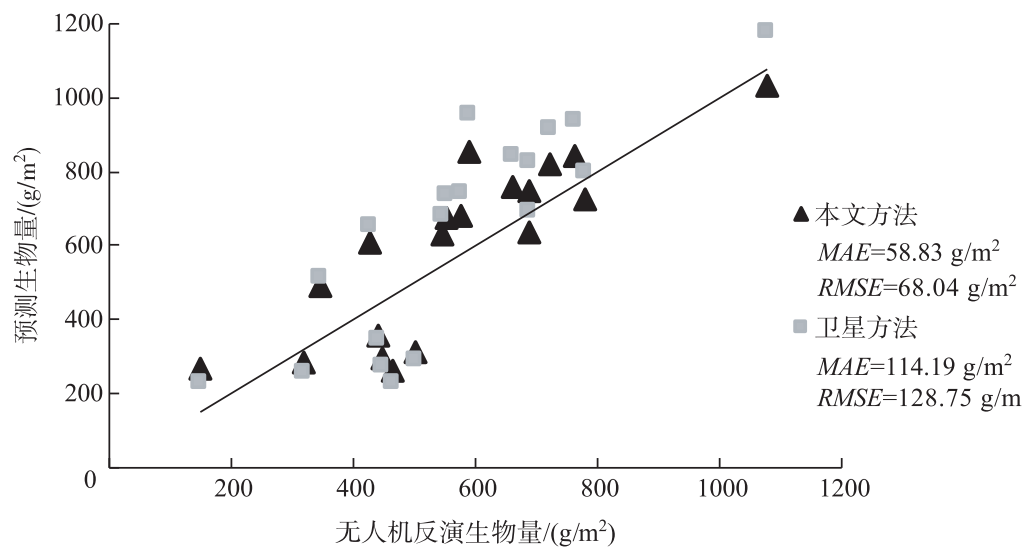

图 8 本文方法与卫星遥感方法生物量反演结果比较

Fig.8 Comparison of biomass inversion results between our method and satellite remote sensing method

\section{4 结论}

本研究将无人机遥感影像、GF-1 WFV 多光谱卫星影像和生物量实测数据相结合, 构建回归模型, 反演 升金湖草滩地上生物量并进行分析. 无人机高空间分辨率的优点可以弥补卫星影像反演生物量时像元真值 难以获取的缺陷, 同时卫星影像可以解决无人机影像生物量反演范围受限的问题. 因此, 本研究针对无人机 可见光波段、可见光植被指数分别建立了 8 种回归模型, 并以 $R^{2} 、 M A E 、 R M S E$ 对反演结果进行分析与评价. 实验结果表明, 在无人机可见光波段中, 基于红波段的湿地地上生物量回归模型具有较好的模拟和预测精 度, 可用于生物量反演; 基于蓝绿波段与基于蓝绿波段植被指数构建的生物量回归模型相关性较差, 地上生 物量反演精度较低.

基于最优回归方程的无人机可见光影像反演, 可以得到与实际情况一致性较好的升金湖保护区湿地地 上生物量分布. 在此基础上, 结合无人机生物量反演结果和 GF-1 WFV NDVI 影像进行回归分析模型构建, 对 升金湖保护区草滩地上生物量进行反演, 并与卫星遥感方法进行定性及定量评价, 结果表明, 本文方法能够 有效提高湿地地上植被生物量反演结果的精度.

通过将无人机和卫星影像相结合进行草滩地上生物量反演, 可以有效提高反演质量, 得到更精确的生 物量空间分布, 且大大减少采样工作量, 降低对调查区域内植被的破坏. 这对于湿地生物量时空变化、影响 因素评价、碳储量反演、湿地恢复等具有重要的应用价值和实际意义. 但由于一些系统误差, 本文方法反演 生物量最低值偏高. 为进一步提高湿地植被地上生物量反演模型精度, 在今后研究中将考虑环境因素, 例如 温度、土壤水分等, 构建多因素反演模型, 收集长时序实测地上生物量样本用以提高模型精度, 对升金湖草 滩植被地上生物量进行时空分析.

\section{5 参考文献}

[ 1 ] Zhao TG, Yu RH, Zhang ZL et al. Estimation of wetland vegetation aboveground biomass based on remote sensing data: A review. Chinese Journal of Ecology, 2016, 35(7) : 1936-1946. DOI:10.13292/j.1000-4890.201607.028. [赵天舸, 于瑞 宏, 张志否等. 湿地植被地上生物量遥感估算方法研究进展. 生态学杂志, 2016, 35(7) : 1936-1946. ]

[ 2 ] Li S, Zhang ZL, Zhou DM. An estimation of aboveground vegetation biomass in a national natural reserve using remote 
sensing. Geographical Research, 2011, 30(2) : 278-290. [李爽, 张祖陆, 周德民. 湿地植被地上生物量遥感估算模 型研究——以洪河湿地自然保护区为例. 地理研究, 2011, 30(2): 278-290.]

[ 3 ] Wang SG, Li X, Zhou YZ. Progress of method for wetland vegetation biomass. Geography and Geo-Information Science, 2004, 20 (5) : 104-109. [王树功, 黎夏, 周永章. 湿地植被生物量测算方法研究进展. 地理与地理信息科学, 2004, $20(5): 104-109$.

[ 4 ] Fan YB, Gong ZN, Zhao WJ et al. Study on vegetation biomass inversion method based on hyperspectral remote sensing. Journal of Hebei Normal University: Natural Science Edition, 2016, 40(3) : 267-271. [范云豹, 宫兆宁, 赵文吉等. 基 于高光谱遥感的植被生物量反演方法研究. 河北师范大学学报: 自然科学版, 2016, 40(3) : 267-271.]

[ 5 ] He C, Feng ZK, Han X et al. The inversion processing of vegetation biomass along yongding river based on multispectral information. Spectroscopy and Spectral Analysis, 2012, 32(12): 3353-3357. [何诚, 冯仲科, 韩旭等. 基于多光谱数据 的永定河流域植被生物量反演. 光谱学与光谱分析, 2012, 32(12) : 3353-3357.]

[ 6 ] Ding L. Biomass and carbon storagr estimation of reed in yellow river estuary wetland based on high resolution remote sensing [Dissertation]. Hohhot: Inner Mongolia University, 2015. [丁蕾. 黄河口湿地芦苇生物量与固碳量高分辨率遥感 估算研究 [学位论文]. 呼和浩特: 内蒙古大学, 2015.]

[ 7 ] Han Y, Pei L, Du J. Remote sensing inversion of aboveground biomass over the honghe wetland. Remote Sensing Technology and Application, 2014, 29(2) : 224-231. [ 韩颖, 裴亮, 杜嘉. 洪河湿地植被地上生物量遥感反演研究. 遥感技术 与应用, $2014,29(2): 224-231$.]

[ 8 ] Wang JB, Zhang J, Ma Y et al. Study on the above ground vegetation biomass estimation model based on GF-1 WFV Satellite Image in the Yellow River Estuary Wetland. Acta Laser Biology Sinica, 2014, 23(6): 604-608. [王建步, 张杰, 马 毅等. 基于高分一号 WFV 卫星影像的黄河口湿地草本植被生物量估算模型研究. 激光生物学报, 2014, 23(6): 604-608.]

[ 9 ] Liang JP, Ma DX, Mao DH et al. Remote sensing based estimation of Phragmites australis aboveground biomass in Shuangtai Estuary National Nature Reserve. Remote Sensing for Land \& Resources, 2016, 28(3) : 60-66. DOI: 10.6046/gtzyyg. 2016.03.10. [梁建平, 马大喜, 毛德华等. 双台河口国际重要湿地芦苇地上生物量遥感估算. 国土资源遥感, $2016,28(3)$ : 60-66. ]

[10] Lumbierres M, Méndez P, Bustamante J et al. Modeling biomass production in seasonal wetlands using MODIS NDVI land surface phenology. Remote Sensing, 2017, 9(4) : 392. DOI: 10.3390/ns9040392.

[11] Wang P, Wang RR, Yang GS. Advance in classification and biomass estimation of plants in wetlands based on multisource remote sensing data. Wetland Science, 2017, 15(1): 114-124. [王鹏, 万荣荣, 杨桂山. 基于多源遥感数据的 湿地植物分类和生物量反演研究进展. 湿地科学, 2017, 15(1): 114-124.]

[12] Wang Q, Liao JJ. Estimation of wetland vegetation biomass in the Poyang Lake area using Landsat IM and ENVISAT ASAR data. Journal of Geo-Informatdn Science, 2010, 12(2) : 282-291. [王庆, 廖静娟. 基于 Landsat TM 和 ENVISAT ASAR 数据的鄱阳湖湿地植被生物量的反演. 地球信息科学学报, 2010, 12(2) : 282-291.]

[13] Zhang B, Zhang L, Xie D et al. Application of synthetic NDVI time series blended from Landsat and MODIS data for grassland biomass estimation. Remote Sensing, 2015, 8(1): 10. DOI: 10.3390/rs801001.

[14] Zhou ZM, Yang YM, Chen BQ. Estimating the Spartina alterniflora fractional vegetation cover using high spatial resolution remote sensing in a coastal wetland. Acta Ecologica Sinica, 2017, 37(2) : 505-512. DOI: 10.5846/stxb201507271566. [周在明, 杨燕明, 陈本清. 滩涂湿地入侵种互花米草植被覆盖度的高空间分辨率遥感估算. 生态学报, 2017, 37 (2) : 505-512.]

[15] Xie T, Liu R, Hu QH et al. A critical review on unmanned aerial vehicle remote sensing technology in the field of environmental monitoring. Environmental Science and Technology, 2013, 26(4): 55-60. [谢涛, 刘锐, 胡秋红等. 基于无人机 遥感技术的环境监测研究进展. 环境科技, 2013, 26(4) : 55-60.]

[16] Zhang ZJ, Li AN, Bian JH et al. Estimating aboveground biomass of grassland in Zoige by Visible Vegetation Index Derived from Unmanned Aerial Vehicle Image. Remote Sensing Technology and Application, 2016, 31(1) : 51-62. [张正健, 李爱农, 边金虎等. 基于无人机影像可见光植被指数的若尔盖草地地上生物量估算研究. 遥感技术与应用, 2016, 31(1) : 51-62.]

[17] He YY, Zhang YB, Li JQ et al. Estimation of stem biomass of individual Abies faxoniana through unmanned aerial vehicle remote sensing. Journal of Bejing Forestry University, 2016, 38(5) : 42-49. DOI: 10.13332/j.1000-1522.20150383. [何 游云, 张玉波, 李俊清等. 利用无人机遥感测定岷江冷杉单木树干生物量. 北京林业大学学报, 2016, 38(5): 42-49.] 
[18] Zhang Z, Sun G, Zhang L et al. Biomass retrieval based on UAVSAR polarimetric data. IEEE, 2010: 604-607. DOl: 10. 1109/IGARSS.2010.5651641.

[19] Jing R, Gong ZN, Zhao WJ et al. Estimating biomass of emergent aquatic plants based on UAV SfM data. Acta Ecologica Sinica, 2017, 37(22) : 7698-7709. DOl: 10.5846/stxb201609221908. [井然, 宫兆宁, 赵文吉等. 基于无人机 SfM 数据的挺水植物生物量反演. 生态学报, 2017, 37(22): 7698-7709.]

[20] Zhou Z, Yang Y, Chen B. Estimating Spartina alterniflora fractional vegetation cover and aboveground biomass in a coastal wetland using SPOT6 satellite and UAV data. Aquatic Botany, 2017, 144. DOI: 10.1016/j.aquabot.2017.10.004.

[21] Han S, He TR, Ban RH. Analysis of ecosystam service function value of Shengj in Lake Wetland. Soil and Water Conservation in China, 2015, (6): 24-27. [韩松, 何太蓉, 班荣舶. 升金湖湿地生态系统服务功能价值分析. 中国水土保 持, 2015, (6): 24-27.]

[22] Yang L, Dong B, Wang Q et al. Habitat suitability change of water birds in Shengjinhu National Nature Reserve, Anhui Province. J Lake Sci, 2015, 27(6): 1027-1034. DOI: 10.18307/2015.0606. [杨李, 董斌, 汪庆等. 安徽升金湖国家 级自然保护区水鸟生境适宜性变化. 湖泊科学, 2015, 27 (6) : 1027-1034.]

[23] Barter M, Cao L, Chen L et al. Results of a survey for waterbirds in the lower Yangtze floodplain, China, in January-February 2004. Forktail, 2005, 21: 1-7.

[24] Wang FL. The bird community in shengjinhu lake wetland and its protection study. Journal of Anqing Teachers College: Natural Science Edition, 2014, 20(2) : 101-104. [汪芳琳. 升金湖湿地保护区鸟类及其保护策略研究. 安庆师范学 院学报：自然科学版, 2014, 20(2): 101-104.]

[25] Yuan XJ. An Introduction on Shengjinhu National Nature Reserve. Anhui Forestry Science and Technology, 2004, (1): 45. [袁西进. 升金湖国家级自然保护区简介. 安徽林业科技, 2004, (1): 45.]

[26] Li X, Sun W, Li L. Study on the recognition of spirulina based on visible light remote sensing of the Small UAV. Geomatics \& Spatial Information Technology, 2017, 40(4): 153-156. [李金金, 孙伟, 李林. 基于小型无人机可见光遥感的蓝藻 识别研究. 测绘与空间地理信息, 2017, 40(4) : 153-156.]

[27] Wang XQ, Wang MM, Wang SQ et al. Extraction of vegetation information from visible unmanned aerial vehicle images. Transactions of the Chinese Society of Agricultural Engineering, 2015, 31(5): 152-159. [汪小钦, 王苗苗, 王绍强等. 基于可见光波段无人机遥感的植被信息提取. 农业工程学报, 2015, 31(5): 152-159.]

[28] Guo P, Wu FD, Dai JG et al. Comparison of farmland crop classification methods based on visible light images of unmanned aerial vehicles. Transactions of the Chinese Society of Agricultural Engineering, 2017, 33(13): 112-119. [ 郭鹏, 武法东, 戴建国等. 基于无人机可见光影像的农田作物分类方法比较. 农业工程学报, 2017, 33(13): 112-119.]

[29] Verrelst J, Schaepman ME, Koetz B et al. Angular sensitivity analysis of vegetation indices derived from CHRIS/PROBA data. Remote Sensing of Environment, 2008, 112(5) : 2341-2353. DOI:10.1016/j.rse.2007.11.001.

[30] Meyer GE. Verification of color vegetation indices for automated crop imaging applications. Elsevier Science Publishers B. V, 2008, 63(2) : 282-293. DOI: 10.1016/j.compag.2008.03.009.

[31] Bendig J, Yu K, Aasen H et al. Combining UAV-based plant height from crop surface models, visible, and near infrared vegetation indices for biomass monitoring in barley. International Journal of Applied Earth Observation \& Geoinformation, 2015, 39: 379-387. DOI: 10.1016/jjag.2015.02.012.

[32] Hunt ER, Cavigelli M, Cst D et al. Evaluation of digital photography from model aircraft for remote sensing of crop biomass and nitrogen status. Precision Agriculture, 2005, 6(4) : 359-378. DOl:10.1007/s11119-005-2324-5.

[33] Wang QY. Comparison between Estimated standard error and coefficient of determination of regression. Statistics and Decision, 2006, (23) : 141. [王巧英. 回归估计标准误差与可决系数的比较. 统计与决策, 2006, (23): 141.]

[34] Li BB, Li ZB, Yu T et al. Research on fractal dimension of vegetation cover based on normalized difference vegetation index in watershed scale. Transactions of the Chinese Society of Agricultural Engineering, 2014, 30(15): 239-247. [李斌 斌, 李占斌, 宇涛等. 基于归一化植被指数的流域植被覆盖分形维数研究. 农业工程学报, 2014, 30(15): 239-247.]

[35] Fu ST, Zhou Y. Research on normalized difference vegetation index algorithm based on remote sensing image. Jiangxi Surveying and Mapping, 2010, (3) : 31-32, 15. [符思涛, 周云. 基于遥感影像的归一化植被指数算法研究. 江西测 绘, $2010,(3): 31-32,15$. 